\title{
Effects of Spin-Isospin-Interactions on Nuclear Collective Motion
}

Takaharu Otsuka a b c, Michio Honma ${ }^{\mathrm{d}}$, and Daisuke Abe ${ }^{\mathrm{a}}$

${ }^{a}$ Department of Physics, University of Tokyo,

Hongo, Bunkyo-ku, Tokyo, 113-0033, Japan

${ }^{\mathrm{b}}$ Center for Nuclear Study, University of Tokyo, Hongo, Bunkyo-ku, Tokyo, 113-0033, Japan

${ }^{\mathrm{c}}$ RIKEN,Hirosawa, Wako-shi, Saitama 351-0198, Japan

${ }^{\mathrm{d} C e n t e r}$ for Mathematical Sciences, University of Aizu, Tsuruga, Ikki-machi, Aizu-Wakamatsu, Fukushima 965-8580, Japan

The effects of the tensor force, the most prominent spin-isospin interaction, will be discussed from the viewpoints of the spherical single-particle energies and the correlation beyond single-particle motions. The former point will be started with basic properties of the tensor force, and a new mean-field model will be introduced. It will be shown that single-particle properties, including (sub-)magic gaps and spin-orbit splittings, are varied rather strongly by the tensor force. The effects are not weaker than the effects of the diffuse surface due to neutron skin. An example is presented for the so-called island of inversion, for which the boundary is being modified significantly. The latter point, correlations beyond single-particle motion, leads us to a special importance of the $N=Z$ nuclei such as ${ }^{56} \mathrm{Ni}$.

\section{Introduction}

The spin-isospin interaction has a special importance for the COMEX conferences as well as their preceding conferences, largely because this interaction is closely connected to spin-isospin excitations, including the Gamov-Teller (GT) resonances. Recently, it has been realized that the spin-isospin interaction, especially the tensor interaction, is also of particular and direct importance to single-particle properties, or mean-field properties, of exotic nuclei. Like other many-body quantal systems, e.g., electrons in hydrogen-like atoms, the mean field governs the structure of nuclei [1]. The deformation, spin-isospin excitation, and even existence of nuclei depend on single-particle properties. Recently it has been shown that the tensor force has a specific, robust and systematic effect on the single-particle energies of nuclei, even breaking or creating magic numbers in some cases [2]. We shall overview the present situation of this subject, and also shall explore perspectives. 


\section{Tensor force and pions}

The nuclear binding is basically due to the exchange of mesons. Even with QCD, this picture remains unchanged. While the QCD may give us more natural explanations of short-distance behaviours, for a longer distance between nucleons, the meson exchange picture is still valid, and its basis may be given by Chiral Perturbation idea of QCD [4].

Yukawa predicted 70 years ago that the binding of atomic nuclei is due to exchange of mesons [3]. We now know that the nuclear binding comes indeed mostly from exchange of more than one meson. This situation can be seen in the importance of the $\sigma$ meson, which is nothing but a pair of pions coupled to spin 0. Besides the effects of multiple meson exchanges, there should be the effect of single pion exchange. It is well-known that the dominant effect of the single-pion exchange is nothing but the tensor force. We have presented [2] that a systematic and significant effect is produced from the one-pion exchange on single-particle energies with many examples over the nuclear chart. The effect can be explaind in a very simple and intuitive way.

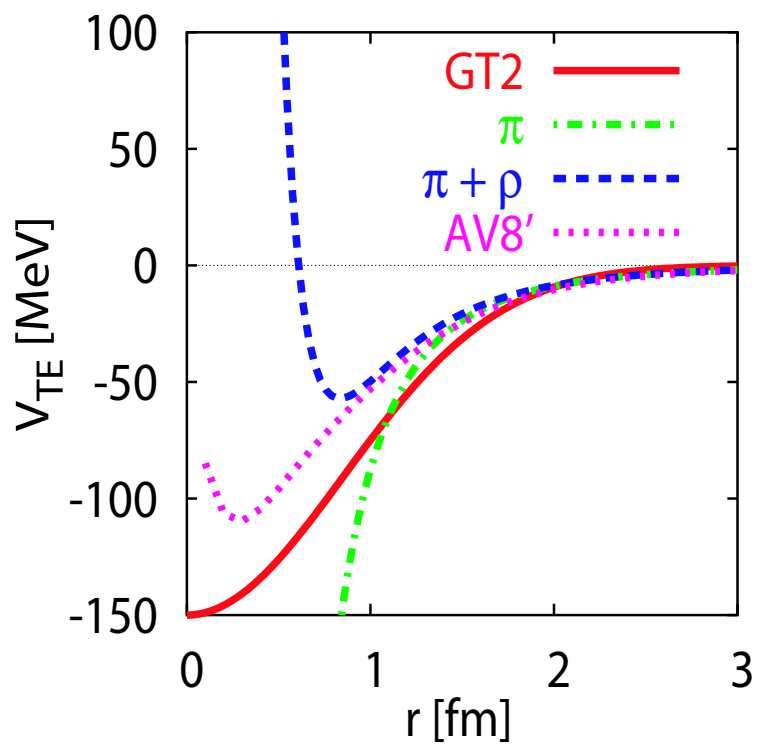

Figure 1. Triplet-Even potential of the tensor force for various potential models.

We here explain that the same tensor force can be expressed in two ways, in order to answer to some questions. The tensor force is expressed usually as,

$V_{T}=\left(\vec{\tau}_{1} \cdot \vec{\tau}_{2}\right) S_{12} V(r)$ 
where $\vec{\tau}_{1,2}$ denotes the isospin operators of nucleons 1 and $2,(\cdot)$ means a scalar product, and $V$ is a function of the distance, $r$, between two nucleons. The function $S_{12}$ is defined as

$S_{12}=3\left(\overrightarrow{s_{1}} \cdot \vec{r} / r\right)\left(\overrightarrow{s_{2}} \cdot \vec{r} / r\right)-\left(\overrightarrow{s_{1}} \cdot \overrightarrow{s_{2}}\right)$,

where $\vec{s}_{1,2}$ is the spin of nucleons 1 and 2 , and $\vec{r}$ denotes relative coordinate from nucleon 1 to 2 with $r$ being its absolute value. This equation is rewritten as

$$
\begin{aligned}
S_{12} & =3\left(\left[\overrightarrow{s_{1}} \times \overrightarrow{s_{2}}\right]^{(2)} \cdot[\vec{r} \times \vec{r}]^{(2)} / r^{2}\right), \\
& =\sqrt{24 \pi / 5}\left(\left[\overrightarrow{s_{1}} \times \overrightarrow{s_{2}}\right]^{(2)} \cdot Y^{(2)}\right)
\end{aligned}
$$

where $[\times]^{(K)}$ means the coupling of two operators in the brackets to an angular momentum (or rank) $K$, and the spherical harmonics is introduced:

$[\vec{r} \times \vec{r}]^{(2)} / r^{2}=\sqrt{8 \pi / 15} Y^{(2)}$.

Thus, the tensor force can be expressed in two different ways. Equation (2) may be more familiar, while eq. (4) is equivalent to it and is used in [2].

Figure 1 shows the so-called Triplet-Even Potential defined from $V(r)$ as

$V_{T E}(r)=-3 V(r)$.

In Fig. 1, the tensor part of the one-pion exchange potential $(\pi)$, the one-pion + one- $\rho$ meson exchange potential $(\pi+\rho)$, AV8' potential [5], and GT2 potential is shown. The tensor part of the AV8' potential is similar to taht of the M3Y potenial [6]. The GT2 potential will be discussed later. One notices similarities among the potentials except for the $\pi$ potential which is shown only for the sake of comparison. Differences of these potentials at short distances do not matter in the present study, because the relative motion between two nucleons cannot be in the $S$ state in both bra and ket states (See [2] for details).

We have investigated the tensor monopole effects using the $\pi+\rho$ potential in the shellmodel based studies [2]. Since the parameters of this potential has been well established by Osterfeld [7], we took the values in [7] without further adjustment. There could be minor higher-order-medium and/or core-polization effects, but they are up to about 10 \%. At moment, we focus on global studies, leaving such fine details for later works.

Since the tensor monopole effects have been discussed extensively in [2], it is not appropriate to repeat the arguments here. We instead mention that more examples of the monopole tensor effects have been seen recently. One of them is for the monopole interaction between proton $1 \mathrm{~d}_{3 / 2}$ and neutron $1 \mathrm{f}_{7 / 2,5 / 2}$ orbits [8]. In fact, the change of the spin-orbit splitting has been measured for the first time in this experiment.

The tensor force should be responsible for the change of the magic number around $N=20$. Figure 2 shows effective single-particle energies (ESPE's) and their changes. The original figure was presented in [9], where the change of the shell structure had been discussed for the first time as a systematic effect of the effective nucleon-nucleon interaction, particularly, its spin-isospin part. It was pointed out in [9] that the monopole interaction between spin-flip proton-neutron partners is crucial. Up to this point, the argument is 


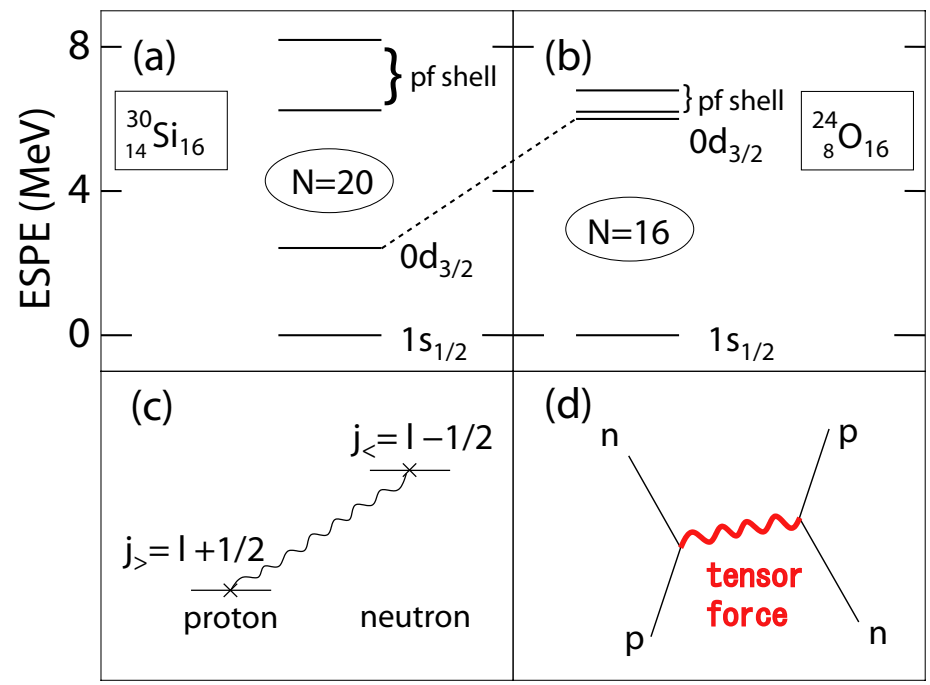

Figure 2. Schematic picture of magicity change due to the spin-isospin interaction. Neutron ESPE's for (a) ${ }^{30} \mathrm{Si}$ and (b) ${ }^{24} \mathrm{O}$, relative to $1 s_{1 / 2}$. The dotted line connecting (a) and (b) is drawn to indicate the change of the $0 d_{3 / 2}$ level. (c) The major interaction producing the basic change between (a) and (b). (d) The process relevant to the interaction in (c) is dominantly due to the tensor force.

still correct. In the same paper, we vaguely attributed the origin of this interaction to the $\sigma \sigma \tau \tau$ central interaction. However, it turns out that the $\sigma \sigma \tau \tau$ central interaction is not the right origin : For instance, it is not strong enough, and if we make it stronger artificially the whole scheme breaks down. We have shown in [2] that the major origin of the shell evolution (change of relative energies of single-particle states as a function of $N$ or $Z$ ) is the tensor force and we can explain the shell evolution without destroying existing descriptions of nuclei. Thus, Fig. 2 differs in the part (d) from the corresponding one in $[9]$.

The $N=20$ shell gap can be small for nuclei with $Z \sim 10$ as we shall show later. This trend has been confirmed by many experiments published recently [10-14], with theoretical explanations obtained with the SDPF-M interaction [15,12].

\section{Correlations beyond mean-field properties}

One of the most recent works on the tensor force is on correlations beyond the meanfield level. This is related also to collective motion. For this purpose, we decomposed the effect of the tensor force for its monopole and multipole parts separately. Figure 3 shows the levels of ${ }^{56} \mathrm{Fe}$ and ${ }^{57} \mathrm{Fe}$ calculated by full pf-shell calculations with MSHELL code [16]. The employed effective interaction is GXPF1A [17], which is a slight revison of GXPF1 


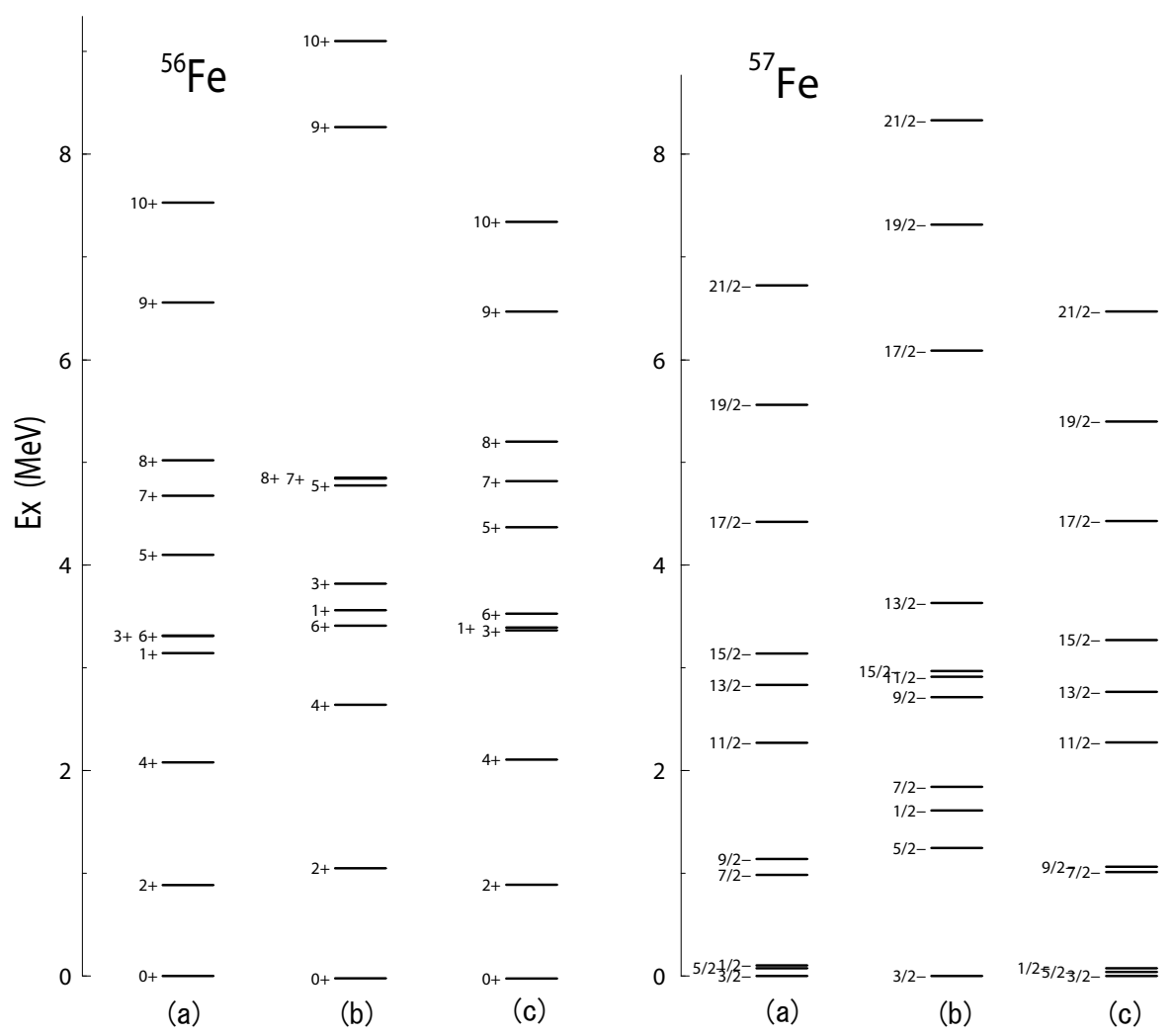

Figure 3. Energy levels of ${ }^{56} \mathrm{Fe}$ and ${ }^{56} \mathrm{Fe}$ calculated with GXPF1A interaction. (a) Full interaction. (b) Tensor component is subtracted. (c) Monopole part of the tensor component is restored to (b).

[18].

Figure 3 (a) (for both ${ }^{56} \mathrm{Fe}$ and ${ }^{57} \mathrm{Fe}$ ) is the result of the calculations using the GXPF1A interaction. In Fig. 3 (b), the tensor contribution to the shell-model interaction is subtracted. The $\pi+\rho$ potential is used. Levels are very different between (a) and (b). We then restore the monopole part of the tensor force to (b), and the results are shown in (c). In other words, only multipole part is subtracted in (c). The results of (c) are not so different from (a), indicating that the monopole part of the tensor force makes crucial contributions to nuclear spectra, wheras multipole part produces much minor contributions. This is very interesting and important. In the conventional pairing+QQ picture, only these pairing and quadrupole degrees of freedom are important. The tensor force is outside, but its effect comes in through effective single-particle energies or monopole changes of single-particle levels.

We then wonder whether this is always true or not. Figure 4 (a) is the result of the 


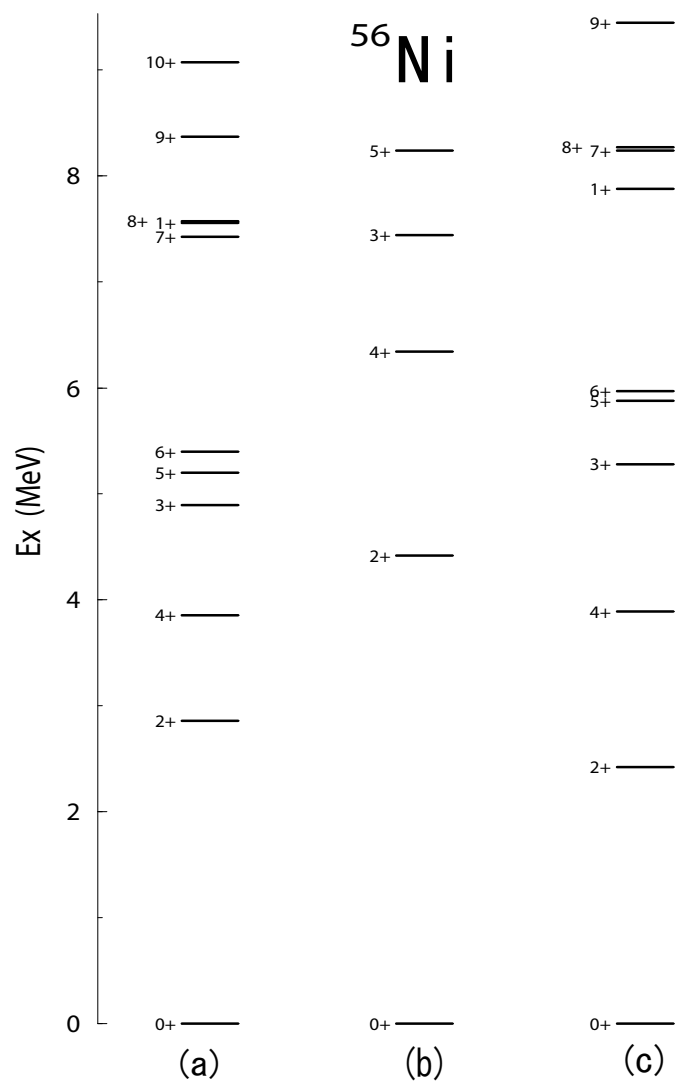

Figure 4. Energy levels of ${ }^{56} \mathrm{Ni}$ calculated with GXPF1A interaction. (a) Full interaction. (b) Tensor component is subtracted. (c) Monopole part of the tensor component is restored to (b).

calculations using the GXPF1A interaction. In Fig. 4 (b), the tensor contribution to the shell-model interaction is subtracted. The $\pi+\rho$ potential is used again. Levels are very different between (a) and (b). We then restore the monopole part of the tensor force to (b), and the results are shown in (c). The results of (c) are still rather different from (a), suggesting that the tensor force contributes not only through the monopole part but also through the multipole part. The difference between (a) and (b) is larger than the cases of ${ }^{56} \mathrm{Fe}$ and ${ }^{57} \mathrm{Fe}$. The difference has been seen also in the Gamov-Teller distribution. Thus, the $N=Z$ nuclei like ${ }^{56} \mathrm{Ni}$ can give us unique information on the nuclear forces. This feature has never been pointed out. This analysis makes sense, partly because the tensor component of the shell-model interaction can be well approximated by the simple $\pi+\rho$ potential, particularly in its $T=0$ channel. Details will be presented elsewhere. 


\section{Mean-field model with tensor force}

The existing Skyrme [19,20] and Gogny [21-23] calculations do not include the tensor force. We have developed a mean-field model with the tensor force. Details are given in $[24]$.

\section{A possible mechanism of /s splitting change}

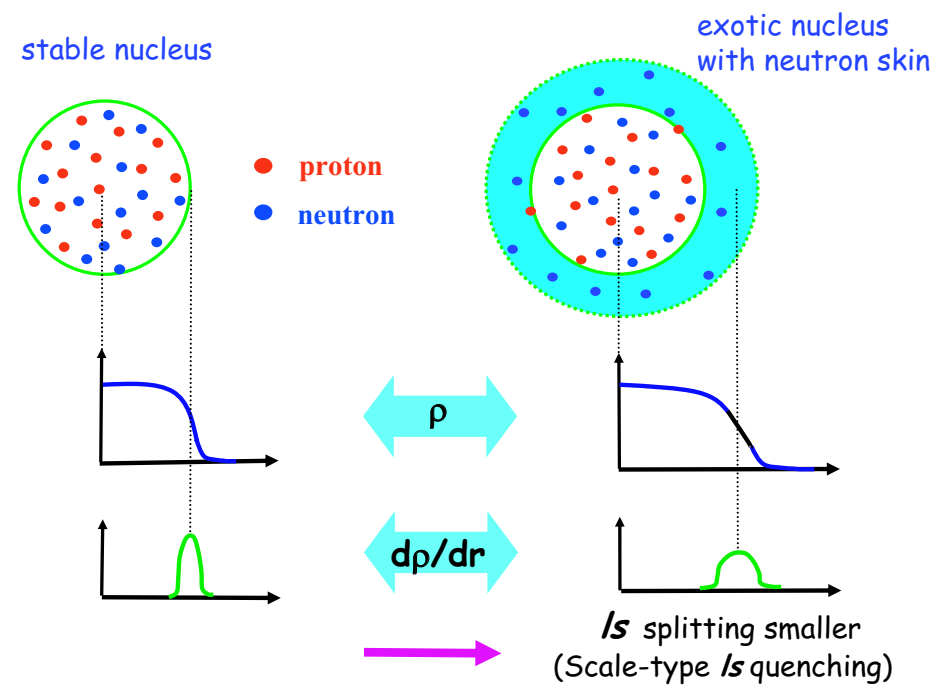

Figure 5. Conventional picture of smaller spin-orbit splitting in neutron-rich nuclei.

The tensor force is expressed as [2],

$V_{T}=\left(\overrightarrow{\tau_{1}} \cdot \overrightarrow{\tau_{2}}\right)\left(\left[\overrightarrow{s_{1}} \overrightarrow{s_{2}}\right]^{(2)} \cdot Y^{(2)}\right) f_{G}(r)$,

Here, $f_{G}(r)$ is a function of the relative distance, $r$. Similarly to the central part of the interaction, we take a Gaussian for $f_{G}(r)$ too, for simplicity, with the range $1.2 \mathrm{fm}$, i.e., the longer range of the central part. The overall strength of $f_{G}(r)$ is then determined so that its volume integral reproduces that of the AV8' [5], resulting in $f_{G}(r=0)=3156$ $(\mathrm{MeV})$. The Triplet-Even potentials in Fig. 1 includes this potential.

One of the major motivations of this work is to compare the effect of neutron skin [26] to the effect of the tensor force. Figure 5 shows the conventional and rather naive picture of the change of the spin-orbit splitting due to more diffuse surface in neutron-rich nuclei. This effect can be a good subject of future experiments as indicated in RIA Physics White Paper [27]. Although the reduction of the splitting may occur very near driplines [28], the reality of the mechanism shown in Fig. 5 should be examined. This mechanism is reffered to as the scale-type ls quenching for the sake of clarification. 


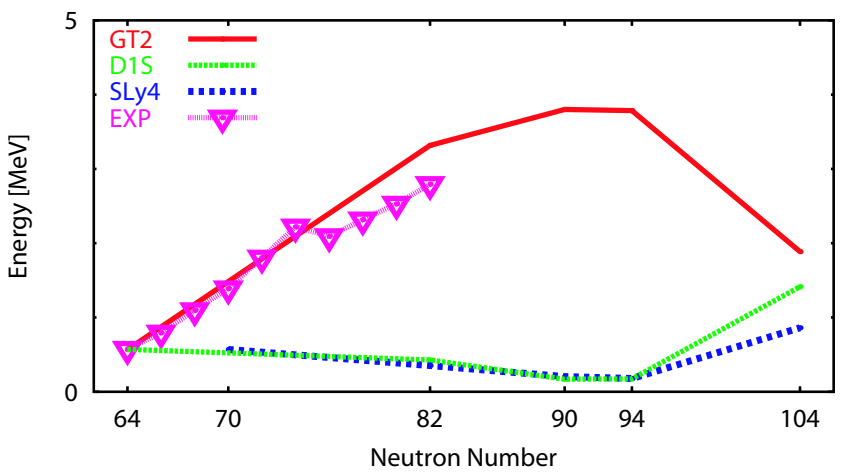

Figure 6. Evolution of $1 h_{11 / 2}-1 g_{7 / 2}$ energy gap. The difference from the value of $N=64$ is plotted for experimental data [29] and calculated results with GT2, D1S and SLy4 interactions.

In [24], we suggested that another type of the quenching of the spin-orbit splitting is the right picture for most (or all) of exotic nuclei accesible at present. Their effects are smaller than the tensor force effect, and in fact the shell evolution cannot be studied without the tensor force. The sysmtematic change of proton single-particle levels of Sb isotopes as shown by Schiffer et al. [29] can be explained with the tensor force, but have not been explained in many attmepts by existing mean-field models. Figure 6 presents such a comparison.

\section{Summary}

In summary, we gave an overview on the evolutions of the nuclear shells. The tensorforce effects are of the same order of magnitude as the changes of spin-orbit splitting due to the neutron skin, and thereby both have to be considered simultaneously. Even if the neutron skin effect is absent or very small, notable tensor effects on SPE's are suggested for examples such as exotic $N=20$ isotones with $Z \sim 10$, exotic Ni isotopes with $N=40 \sim 50$, and a long isotopic chain of Sb. Note that many of interesting physics cases are found well inside the dripline, but quite far away from the $\beta$-stability line. The predictions seem to provide us with a paradigm to be tested and developed by future RI-beam physics. We stress that changes in SPE's affect essentially all features of the nuclear structure, e.g., deformation, and also that many of the nuclei involved participate to the astrophysical $r$-process most likely. We point out that a large fraction of the tensor force comes from one $\pi$ exchange, and the present GT2 model includes this mechanism. So, the question is "Are pions so visible in exotic nuclei ?".

The tensor effect beyond the spherical mean-field level is presented. For $N=Z$ nuclei like ${ }^{56} \mathrm{Ni}$, this is particularly important and interesting.

The $l s$ quenching has two types. The surface type has been expected [27] but does 
not appear in nuclei studied in this work. The situation could be different if the nucleus sits on or very near the dripline [28]. The position type, which has not been reported in publications, can be found in many cases.

This work was supported in part by a Grant-in-Aid for Specially Promoted Research (13002001) from the MEXT and by the JSPS Core-to-Core Program. This work has been a part of the RIKEN-CNS joint research project on large-scale nuclear-structure calculations.

\section{REFERENCES}

1. P. Ring and P. Schuck, The Nuclear Many-Body Problem, (Springer, Berlin, 1980).

2. T. Otsuka, T. Suzuki, R. Fujimoto, H. Grawe and Y. Akaishi, Phys. Rev. Lett. 95, 232502 (2005).

3. H. Yukawa, Proc. Phys. Math. Soc. Japan 1748 (1935).

4. S. Weinberg, Phys. Lett. B 251, 288 (1990).

5. B.S. Pudliner et al., Phys. Rev. C56, 1720 (1997).

6. G. Bertsch, J. Borysowicz, H. McManus and W.G. Love, Nucl. Phys. A284, 399 (1977).

7. F. Osterfeld, Rev. Mod. Phys. 64, 491(1992).

8. O. Sorlin et al., Phys. Rev. Lett. 97, 92301 (2006).

9. T. Otsuka et al., Phys. Rev. Lett. 87, 082502 (2001).

10. E. Becheva, et al., Phys. Rev. Lett. 96, 012501 (2006).

11. M. Belleguic et al., Phys. Rev. C72, 054316 (2005).

12. Y. Utsuno et al., Phys. Rev. C70, 044307 (2004).

13. V. Tripathi, et al., Phys. Rev. Lett. 94, 162501 (2005).

14. G. Neyens, et al., Phys. Rev. Lett. 94, 022501 (2005).

15. Y. Utsuno et al., Phys. Rev. C60, 054315 (1999).

16. T. Mizusaki, RIKEN Accel. Prog. Rep. 3314 (2000).

17. M. Honma et al., Euro. Phys. J. A25, Suppl. 1, 499 (2005).

18. M. Honma et al., Phys. Rev. C65, 061301 (2002); Phys. Rev. C68, 034316 (2003).

19. T.H.R. Skyrme, Nucl. Phys. 9, 615 (1959).

20. D. Vautherin and D.M. Brink, Phys. Rev. C5, 626 (1972).

21. J. Decharge, M. Girod and D. Gogny, Phys. Lett. 55B, 361 (1975).

22. J. Decharge and D. Gogny, Phys. Rev. C21, 1568 (1980).

23. J.F. Berger, M. Girod and D. Gogny, Nucl. Phys. A428, 23c (1984); Comput. Phys. Commun. 63, 365 (1991),

24. T. Otsuka, T. Matsuo and D. Abe, Phys. Rev. Lett. accepted.

25. Fl. Stancu, D.M. Brink and H. Flocard, Phys. Lett. 68B, 108 (1977).

26. N. Fukunishi, T. Otsuka and I. Tanihata, Phys. Rev. C48, 1648 (1993).

27. RIA Physics White Paper.

28. J. Dobaczewski et al., Phys. Rev. Lett. 72, 981 (1994).

29. J.P. Schiffer et al., Phys. Rev. Lett. 92, 162501 (2004). 